\title{
Regionalisierung wider Willen? Die EU-Regionalpolitik und das „Europa der Regionen“
}

Kann die Regionalisierung von Mitgliedstaaten der Europäischen Union als ein Effekt europäischer Regionalpolitik begriffen werden? Diese Frage stellt sich mit Blick auf die „,alten “, aber auch die zum 1. Mai 2004 der EU beigetretenen Staaten. Seit der Einheitlichen Europäischen Akte von 1987 ist die ursprüngliche „Blindheit“ der europäischen Integration gegenüber den Regionen gewichen. Gleichwohl blieb die Aufwertung der Regionen im Vertragswerk eng begrenzt. Eine differenzierte Analyse des Einflusses, den die Regionen im Politikzyklus der Regionalpolitik gegenüber zentralstaatlichen Akteuren und der Europäischen Kommission ausüben können, offenbart, dass die ,goldenen Zügel“ der EU-Regionalpolitik nur in begrenztem Umfang zur rechtlichen, politischen und finanziellen Aufwertung der Regionen führen. Zwar forcieren selbst zentralistisch ausgerichtete Staaten die Dezentralisierung ihrer Verwaltungen. Als Effekt der EU-Regionalpolitik lassen sich auch regionalpolitische Belange durch die Akteure auf regionaler Ebene besser artikulieren und gegen Widerstand der zentralstaatlichen Ebene wirksamer durchsetzen. Aber eine staats- und verfassungsrechtliche Aufwertung der Regionen kommt gegen den Willen der zentralstaatlichen Ebene nicht zustande. Die Mitgliedstaaten widersetzen sich weiterhin einer ihren Interessen zuwiderlaufenden Regionalisierung im Inneren ihrer Staatsgebilde.

Inhalt

1. Einleitung 458

2. Region - ein unbestimmter Begriff 458

3. Die Regionen im Primärrecht der EU - von der Einheitlichen Akte bis zum Verfassungsvertrag 461

a) Einheitliche Europäische Akte, EEA (1. Juli 1987 in Kraft getreten) 461

b) Vertrag von Maastricht (1. November 1993 in Kraft getreten) 462

c) Vertrag von Amsterdam (1. Mai 1999 in Kraft getreten) 463

d) Vertrag von Nizza (1. Februar 2003 in Kraft getreten) 463

e) Vertrag über eine Verfassung für Europa

(18. Juni 2004 unterzeichnet) 
4. Die Rolle der Regionen in der EU-Regional- und Strukturpolitik 466

5. Der Einfluss von Regionen auf die Gestaltung der Strukturpolitik 468

6. Die Beteiligung der Regionen in der Politikphase der Programmplanung 471

7. Widersprüchliche Regionalisierungstendenzen in zentralisierten Mitgliedstaaten der EU

a) Frankreich 474

b) Vereinigtes Königreich 475

c) Griechenland 476

d) Polen 477

e) Ungarn 478

8. Fazit: Regionalisierung wider Willen? 479

Literatur 482

\section{Einleitung}

Zum 1. Mai 2004 ist die Europäische Union um zehn neue Mitgliedstaaten erweitert worden. Gemäß den Kriterien von Kopenhagen aus dem Jahr 1993 mussten die Beitrittsländer nicht nur funktionierende Demokratien und Marktwirtschaften aufweisen. Sie mussten auch den gesamten rechtlichen Besitzstand (acquis communautaire) der EU übernehmen. Damit sollte sichergestellt werden, dass die Gemeinschaftspolitiken auch in den neuen Mitgliedsstaaten angewendet werden. Unter den EU-Politiken war die europäische Struktur- und Regionalpolitik wegen der umfangreichen Finanzmittel für die Neumitglieder von besonderem Interesse. Um in den Genuss dieser Mittel zu gelangen, müssen die Mitgliedstaaten über regionale Gebietseinheiten verfügen. Wo diese nicht vorhanden waren, mussten sie in Vorbereitung auf den Beitritt eingerichtet werden. Da die Transformationsstaaten in Mittel- und Osteuropa traditionell zentralistisch ausgerichtet waren, stellt sich die Frage, inwieweit die EU-Regionalpolitik Prozesse zur Regionalisierung in den neuen Mitgliedstaaten begünstigt. Ist es vielleicht da, wo die zentralstaatlichen Autoritäten sich gegen eine Abgabe von Kompetenzen gesperrt haben, zu einer „Regionalisierung wider Willen“ gekommen? Und hat die Regionalpolitik dem viel beschworenen „Europa der Regionen“ Auftrieb gegeben?

$\mathrm{Da}$ in etlichen Transformationsstaaten die Regionalisierung von den Titularnationen mit der Gefahr des Separatismus, oder gar der Sezession in Verbindung gebracht wird, erhalten die Fragestellungen auch eine erhebliche politische Brisanz. Auf diesen Aspekt kann allerdings nicht näher eingegangen werden.

\section{Region - ein unbestimmter Begriff}

Region - das ist ein geläufiger, gleichwohl selten präzise bestimmter Begriff. Eine Definition fällt schwer. Zu Recht verweist Keating darauf, dass recht unterschiedliche Aspekte relevant sind, wenn man Regionen in ihrer gliedstaatlichen Dimension betrachtet: institutionelle Strukturen gehören ebenso dazu wie Fähigkeiten zur aktiven Politikgestaltung, 
Kompetenzbestände, integrative Potenziale, finanzielle Ressourcen, Ausgestaltung intergouvernementaler Beziehungen innerhalb regionalisierter oder föderaler Ordnungen sowie die Einbindung in das Marktgeschehen. Region könne daher nicht bloß als eine „Ebene“ in der territorialen Hierarchie verstanden werden, weil die Macht in Netzwerken und vielfältigen Sphären der Autorität verteilt sei (Keating 1998).

Begnügt man sich mit einer heuristischen Definition, dann kann man auf die Versammlung der Regionen Europas (VRE) zurückgreifen, die in Art. 2 ihrer Satzung Region wie folgt bestimmt: „Als ,Regionen' gelten grundsätzlich jeweils die unmittelbar unterhalb der zentralstaatlichen Ebene bestehenden Gebietskörperschaften mit einer politischen Vertretung, welche von einer gewählten regionalen Versammlung wahrgenommen wird“ (Versammlung der Regionen Europas 2002). Mit Hrbek und Weyand werden die Regionen als ,diejenigen territorialen Einheiten [...] bezeichnet, die unmittelbar unterhalb der Zentralregierung, aber über der kommunalen Ebene angesiedelt sind, die für die territoriale Organisation der Verwaltung von Bedeutung sind und in deren Rahmen Entscheidungsträger, die nicht unmittelbar dem Zentralstaat angehören, bestimmte Aufgaben wahrnehmen“" (1994: 18 f.). Bezieht man diese Definition auf die EU, dann wird diese als dreigliedriges Mehrebenensystem mit einer supranationalen, nationalen und subnationalen, eben regionalen Ebene erkennbar. ${ }^{1}$ Das viel zitierte „Europa der Regionen“ impliziert die Existenz dieses Mehrebenensystems, das in der wissenschaftlichen Literatur aus guten Gründen fast immer mit dem Adjektiv „,dynamisch“ verbunden wird, um zu verdeutlichen, dass die EU keineswegs ihren finalen Zustand erreicht hat, sondern sich als ein „Gebilde sui generis“" in einem Entwicklungsprozess befindet. Auch die Regionen dürften deshalb in diesem Gebilde noch nicht ihren endgültigen Platz gefunden haben.

Wenn im Folgenden von Regionalpolitik die Rede ist, dann sind spezifische Maßnahmen zum Ausgleich regionaler Ungleichgewichte (Disparitäten) angesprochen. Strukturpolitik ist demgegenüber der umfassendere Begriff. Er schließt die Regionalpolitik ein, erfasst aber zusätzlich auch Maßnahmen, mit denen z. B. das Humankapital in allen Mitgliedstaaten der EU gefördert und entwickelt wird. ${ }^{2}$ Mit der Systematik der NUTS (Nomenclature des Unités Territoriales Statistiques) hat die EU eine dreigliedrige Einteilung von Gebietseinheiten vorgenommen (Eurostat Regionen 1995). Die Gebietseinheiten wer-

1 Auf die kommunale Ebene, die in der EU die „vierte Ebene“ etabliert, wird im Folgenden nicht weiter eingegangen.

2 Die sektorale Strukturpolitik bezieht sich auf einzelne Industrien oder Branchen und fördert deren Entwicklung und Anpassung an globale Wettbewerbsbedingungen. Die regionale Strukturpolitik, auch Regionalpolitik genannt, fördert gezielt bestimmte Regionen, um deren wirtschaftliches Niveau und Lebensstandard anzuheben. In Ergänzung richtet sich die horizontale Strukturpolitik nicht an bestimmte Wirtschaftssektoren oder Regionen, sondern begünstigt gleichermaßen alle Wirtschaftssubjekte. Das gilt z. B. für weite Bereiche der Infrastrukturpolitik, weil durch die Verbesserung der Verkehrsverbindungen, der Telekommunikation, der Energieversorgung, der Bildungseinrichtungen, der Gesundheitsfürsorge, der Sozialversicherung u. a. m. die Ausgangsbedingungen für wirtschaftliche Entwicklung und Wohlfahrt verbessert werden. Horizontale Strukturpolitik bezieht sich auch auf das Humankapital, indem die Bildung, die Aus- und Weiterbildung verbessert und Arbeitskräfte gezielt qualifiziert werden (Axt 2000a: 19 f.). 
den von den Mitgliedstaaten vorgeschlagen und von der Kommission genehmigt. Man unterscheidet danach die Ebenen I bis III. Dabei entsprechen die Gebietseinheiten:

- NUTS I den 75 Regionen in der EU-15 (in Deutschland den Ländern) ${ }^{3}$,

- NUTS II den 206 Verwaltungseinheiten in der EU-15 (in Deutschland den Regierungsbezirken) und

- NUTS III den Untergliederungen von Ebene II (in Deutschland den Kreisen).

Obwohl angestrebt wird, dass die Gebietseinheiten hinsichtlich Größe und Bevölkerungsstruktur in etwa vergleichbar sind, weisen einige Regionen doch erhebliche Unterschiede untereinander auf. Die französische Region Ile de France hat z. B. eine Einwohnerzahl von 11 Mio., während es bei der Region Wales im Vereinigten Königreich lediglich 2,9 Mio. sind. Die NUTS-Einheiten erfassen keine zusammenhängenden Wirtschaftsräume, ökonomische Verflechtungen bleiben oftmals außer Betracht. Das politische Gewicht der Gebietseinheiten ist sehr unterschiedlich. Es finden sich darunter Einheiten mit Gesetzgebungskompetenzen (deutsche Länder), aber auch solche, die weitgehend von der Zentralregierung (Frankreich, Großbritannien) abhängig sind.

Die Gebietseinheiten nach der NUTS-Systematik spielen im Rahmen der europäischen Regionalpolitik eine wichtige Rolle. Für die EU-Regionalpolitik sind die NUTS IIEinheiten von zentraler Bedeutung. Dafür, dass EU-weit die Territorien der Mitgliedstaaten in Gebietseinheiten untergliedert werden, sind drei Gründe maßgeblich:

Erstens sind sozio-ökonomische Daten auf der Ebene der Regionen erforderlich, um zu beurteilen, ob sich Gebietseinheiten zur Förderung durch die Regionalpolitik qualifizieren. Diese Daten müssen nach den Vorgaben der europäischen Statistik (EUROSTAT) miteinander vergleichbar sein. Bei Ziel 1 muss z. B. eine Gebietseinheit ein Bruttoinlandsprodukt (BIP) pro Kopf von unter $75 \%$ des EG-Durchschnitts aufweisen, um eine Förderung zu erhalten.

Zweitens bedarf es eigenständiger Administrationen auf regionaler Ebene, um - teilweise in Zusammenarbeit mit den nationalen Organen - Entwicklungspläne auszuarbeiten, diese mit der Europäischen Kommission zu erörtern und die genehmigten Programme schließlich zu implementieren.

Drittens sind Regionen Empfänger von regionalpolitischen Subventionen aus den europäischen Strukturfonds.

Weil die neuen Mitgliedstaaten das System der NUTS zu übernehmen hatten, ist zu prüfen, ob damit eine Regionalisierung in diesen Staaten einhergegangen ist. Wenn man von Regionen spricht, muss man die Heterogenität im Staatsaufbau der Mitgliedstaaten der EU berücksichtigen. Regionen stellen sich ganz unterschiedlich dar, je nachdem ob es sich um föderale oder aber Zentralstaaten handelt (Föhn 2003: 15). Man kann unterscheiden: föderale Staaten (Deutschland, Belgien, Österreich), regionalisierte bzw. dezentralisierte Staaten (Italien, Spanien, Niederlande, Portugal) und zentrale Einheitsstaaten (Dänemark, Frankreich, Finnland, Griechenland, Großbritannien, Irland, Luxemburg, Schweden). ${ }^{4}$

3 In den neuen EU-Staaten fallen hierunter die gesamten Staatsterritorien.

4 Die zum 1. Mai 2004 beigetretenen Staaten Mittel- und Osteuropas gehören aufgrund ihrer vormals sozialistischen Prägung der letzten Gruppe an (vgl. Abschnitt 7). 


\section{Die Regionen im Primärrecht der EU - von der Einheitlichen Akte bis zum Verfassungsvertrag}

Die europäische Gemeinschaftsbildung hatte ursprünglich keinen Platz für die Regionen. Sie war gegenüber den Regionen ,blind“.5 Geht man von den mit der EGKS und später der Europäischen Wirtschaftsgemeinschaft (EWG) und Europäischen Atomgemeinschaft (EURATOM) geschaffenen Organen aus, dann kannte das sich integrierende Europa lediglich zwei Ebenen: die nationale und die supranationale. Die subnationale Ebene war in die Integration nicht einbezogen. Regionen sucht man in den Gründungsverträgen vergeblich. Das hat sich heute geändert. Betrachtet man die Organe und Einrichtungen der EU nach heutigem Stand, stoßen wir auf den Ausschuss der Regionen (AdR). Die Regionen haben sich offensichtlich emanzipiert.

Die europäische Integration ist ein dynamischer Prozess. Das betrifft mehrere Aspekte: Erstens hat sich der materielle Gehalt der Integration auf immer mehr Politikfelder ausgedehnt. Ging es ursprünglich lediglich um einen gemeinsamen Markt für Kohle- und Stahlprodukte (EGKS 1952), so hat sich diese sektorale Integration innerhalb weniger Jahre zu einem gesamtwirtschaftlichen Gemeinsamen Markt (EWG 1958) erweitert. Bis heute sind weitere Politikfelder einbezogen worden. Exemplarisch sei verwiesen auf die Struktur-, die Forschungs-, die Umweltpolitik, aber auch die Gemeinsame Außen- und Sicherheitspolitik sowie die Innen- und Justizpolitik. Zweitens hat sich das Gewicht der europäischen Rechts- und Gesetzgebung verstärkt. Man schätzt, dass heute mehr als $70 \%$ der vom Bundestag beschlossenen Gesetze auf Vorgaben der EU beruhen. Drittens hat sich im Zuge dieser Entwicklung die Bedeutung einzelner EU-Organe verändert. Das Europäische Parlament hat sich z. B. von einer bloß beratenden Versammlung, in die Abgeordnete aus den nationalen Parlamenten entsandt wurden, immer mehr zu einem echten Legislativorgan entwickelt, wenn es um den vergemeinschafteten Bereich der EU (die so genannte 1. Säule) geht. Was sich vom Europäischen Parlament sagen lässt, trifft im Grundsatz auch für die Regionen zu: Im Primärrecht der EU (Verträge) ist im Laufe der Zeit ihre Bedeutung anerkannt worden, und mit den Vertragsänderungen sind die Mitwirkungsmöglichkeiten erweitert worden.

\section{a) Einheitliche Europäische Akte, EEA (1. Juli 1987 in Kraft getreten)}

Die EEA geht zwar nicht auf die Regionen ein, sie enthält als wesentliche Bestimmungen vielmehr die Vollendung des Binnenmarkts bis Ende 1992, die Einfügung des Europäischen Rats in das Primärrecht, die Einführung des Zusammenarbeitsverfahrens mit der Aufwertung des legislativen Bedeutung des Europäischen Parlaments, die Ergänzung des EG-Vertrags um die Sozial-, Struktur-, Forschungs-, Technologie- und Umweltpolitik sowie die verbesserte Zusammenarbeit in der Außenpolitik (Europäische Politische Zusam-

5 Engel spricht in diesem Zusammenhang mit Bezug auf Deutschland von der „Landes-Blindheit“ der EG-Verträge (1994: 92). 
menarbeit, EPZ). Die Anreicherung der EG-Kompetenzen rief aber die Regionen auf den Plan. Besonders die deutschen Länder taten sich hervor. Diese besitzen Staatscharakter und befürchteten, dass ihre Kompetenzen durch die EG ausgehöhlt würden. Innerstaatlich erreichten sie schließlich eine verstärkte Beteiligung, wenn es um EG-Angelegenheiten geht (Bundesratsverfahren nach Art. 2 des Ratifikationsgesetzes zur Einheitlichen Europäischen Akte, EEAG von 1986). Der Bundesrat richtete daraufhin eine EG-Kammer ein, um die EG-Vorlagen bearbeiten zu können. Man kann die diesbezüglichen Aktivitäten der deutschen Länder anlässlich der EEA-Debatte durchaus als Ouvertüre der zunehmend selbstbewusster auftretenden Regionen interpretieren.

\section{b) Vertrag von Maastricht (1. November 1993 in Kraft getreten)}

Kann man in Bezug auf die EEA davon sprechen, dass die deutschen Länder eine gewisse Pionierrolle übernommen haben, um die Rechte der Regionen zu stärken, so gilt das erst recht für den Maastricht-Vertrag. Aus Ländersicht war das nachvollziehbar, sollten doch im EUVertrag Kernkompetenzen der Länder aus dem Bereich der Innen- und Justizpolitik mit der „,dritten Säule“ der EU in die europäische Verantwortung überführt werden - freilich in der recht schwachen Form der intergouvernementalen und nicht der supranationalen Kooperation. Gemeinsam mit anderen europäischen Regionen gelang es schließlich, die Regionen stärker im Primärrecht der EU zu verankern. Als Erfolge für die Regionen sind vorrangig zu nennen:

- Mit der Verankerung des Subsidiaritätsprinzips im EG-Vertrag (Art. 3b, aktuell Art. 5 des EG-Vertrags) soll verhindert werden, dass die Übertragung von Zuständigkeiten an die EG in Bereichen, wo nicht die ausschließliche Zuständigkeit der Gemeinschaft gegeben ist, zulasten der nationalen und subnationalen Ebene (Regionen) erfolgt.

- Mitgliedsstaaten können sich nach Art. 146 EG-Vertrag (Art. 203 aktuell) auch durch Vertreter von Regionen im Rat vertreten lassen, sofern diese den Rang eines Ministers haben und befugt sind, für die Regierung des Mitgliedstaates verbindlich zu handeln.

- Mit dem Ausschuss der Regionen wurde auf EG-Ebene eine Einrichtung geschaffen, die sich aus Vertretern der regionalen, aber auch lokalen Körperschaften zusammensetzt (Art. 198a EG-Vertrag in der Fassung des Maastricht-Vertrags). Der Ausschuss hat zwar eine Aufwertung der Regionen gebracht, genießt jedoch nicht den Status eines „Organs“ (wie Parlament, Kommission, Rat, Gerichtshof und Rechnungshof), was vor allem darin zum Ausdruck kommt, dass er lediglich eine beratende Funktion hat. In bestimmten Fällen muss der Ausschuss von den EG-Organen gehört werden. Das ist vor allem der Fall, wenn regionale oder lokale Angelegenheiten und Kompetenzbereiche betroffen sind. In anderen Fällen kann er von sich aus eine Stellungnahme abgeben. An der Entscheidungsfindung ist der Ausschuss nicht beteiligt. Eine Rechenschaftspflicht der Organe, wie sie mit den Vorschlägen des Ausschusses umgegangen sind, besteht nicht. Immerhin ist mit dem Ausschuss aber auch im europäischen Primärrecht anerkannt worden, dass den Regionen Gehör zu schenken ist und dass sie - wenn auch in einer eher schwachen Form - an Entscheidungen auf europäischer Ebene zu beteiligen sind. 
Hatten die Regionen damit einen „Fuß in der Tür“ europäischer Willensbildungsprozesse, so konnten sie sich gleichwohl nicht mit der Forderung nach einem eigenen Klagerecht vor dem Europäischen Gerichtshof durchsetzen.

\section{c) Vertrag von Amsterdam (1. Mai 1999 in Kraft getreten)}

Bei der Beratung des Vertrags von Amsterdam versuchten die Regionen erneut, ein Klagerecht zu Ihren Gunsten durchzusetzen, blieben dabei aber wie zuvor beim Maastricht-Vertrag erfolglos. Erfolge konnten dagegen in drei wichtigen Fragen erzielt werden: Der mit dem Maastricht-Vertrag verankerte Subsidiaritäts-Artikel wurde in einem eigenen Protokoll (Nr. 30) ausführlich präzisiert. Es wird nunmehr u. a. verlangt, dass die Kommission jeden Vorschlag für einen gemeinschaftlichen Rechtsakt gemäß qualitativen und auch quantitativen Kriterien zu begründen hat. ${ }^{6}$ Der Ausschuss der Regionen bekam einen eigenen Verwaltungsunterbau und musste sich diesen nicht länger mit dem Wirtschafts- und Sozialausschuss teilen. Die Regionen und allen voran die deutschen Länder waren bei dem Bestreben erfolgreich, den Bereich der öffentlichen Daseinsvorsorge und das System der öffentlich-rechtlichen Kreditinstitute (vorerst) vor dem Zugriff europäischer Gesetzgebung zu schützen. ${ }^{7}$

\section{d) Vertrag von Nizza (1. Februar 2003 in Kraft getreten)}

Da der Vertrag von Amsterdam institutionelle Fragen für die erweiterte EU offen gelassen hatte, kam es im Februar 2000 zu einer Revisionskonferenz, die diese „left overs“ regeln sollte. Die Regionen brachten wiederum ihre Anliegen vor, darunter vor allem das Klagerecht, das Fragerecht gegenüber der Kommission, die Sicherung der kommunalen Selbstverwaltung und der öffentlichen Daseinsvorsorge sowie einen Katalog zur Abgrenzung der Kompetenzen zwischen Union, Mitgliedstaaten und Regionen. So wie der Vertrag insgesamt wenig befriedigte, weshalb eine erneute Erörterung der Thematik im Rahmen des Konvents zum Europäischen Verfassungsvertrag für erforderlich gehalten wurde, fiel das Ergebnis auch für die Regionen enttäuschend aus. Lediglich zwei Neuerungen sind erwähnenswert: Zum ersten wurde die notwendige Qualifikation der Mitglieder des Ausschusses der Regionen präzisiert. Danach müssen diese ,entweder ein auf Wahlen beruhendes Mandat in einer regionalen oder lokalen Gebietskörperschaft innehaben oder gegenüber einer gewählten Versammlung politisch verantwortlich“" sein (Art. 263 EG-

6 Die föderal organisierten Mitgliedstaaten Deutschland, Österreich und Belgien haben in einer von der Regierungskonferenz zur Kenntnis genommenen Erklärung (Nr. 3) erklärt, dass die Vorkehrungen im Zusammenhang mit dem Subsidiaritätsprinzip nicht nur die Mitgliedstaaten, sondern auch deren Gebietskörperschaften betreffen, ,,soweit diese nach nationalem Verfassungsrecht eigene gesetzgeberische Befugnisse besitzen“.

7 Vgl. die von der Regierungskonferenz angenommene Erklärung (Nr. 37) zu öffentlich-rechtlichen Kreditinstituten in Deutschland. 
Vertrag). Zum zweiten wurde im Vorfeld der anstehenden EU-Erweiterung um bis zu zwölf neue Mitgliedstaaten festgelegt, wie viele Sitze auf die neuen Mitglieder entfallen. ${ }^{8}$ Darüber hinaus regelt Art. 263 EG-Vertrag, dass die Zahl der Mitglieder im Ausschuss auf 350 begrenzt wird.

\section{e) Vertrag über eine Verfassung für Europa (18. Juni 2004 unterzeichnet)}

Beim Abschluss des Nizza-Vertrags war man sich bereits darüber einig, dass dieses Vertragswerk zwar Minimalregeln für die anstehende Erweiterung der EU festgelegt hatte, dass aber weitere Reformschritte in Richtung einer Vertiefung der Integration notwendig waren. In einer dem Vertrag beigefügten Erklärung (Nr. 20) zur Zukunft der Union wurde ein Prozedere vereinbart, wonach unter Beteiligung der EU-Organe, der nationalen Parlamente und der Öffentlichkeit über die Zukunft der EU debattiert werden sollte. Dabei sollte es um Transparenz und Legitimation ebenso gehen wie um Vereinfachung der Verträge und Festlegung der Rolle der nationalen Parlamente. Für die Regionen besonders bedeutsam, wurde festgelegt, dass eine ,genauere, dem Subsidiaritätsprinzip entsprechende Abgrenzung der Zuständigkeiten zwischen der Europäischen Union und den Mitgliedstaaten" erreicht werden sollte.

Die Regionen beteiligten sich aktiv an der Debatte. Seitens der deutschen Länder wurde eine umfangreiche Liste mit Forderungen vorgelegt. Es ging dabei u. a. um das Klagerecht der Regionen mit Gesetzgebungskompetenz, um das Klagerecht des Ausschusses der Regionen, die Ablehnung der „Methode der offenen Koordinierung ${ }^{\text {"99 }}$, die Ablehnung von Gesetzgebungskompetenzen der EU in Bereichen der Daseinsvorsorge, die klare Abgrenzung der Kompetenzen, die Präzisierung des Subsidiaritätsprinzips und der Verhältnismäßigkeit, die Schaffung eines diesbezüglichen Frühwarnsystems für nationale Parlamente und den Ausschuss der Regionen (Bauer 2004: 458 f.). Im Zuge der Konventverhandlungen konnten einige der genannten Forderungen im Verfassungsvertrag verankert werden:

- Der Verfassungstext würdigt ausdrücklich und erstmalig die Regionen, heißt es doch in Art. I-5, dass die Union nicht nur die nationale Identität der Mitgliedstaaten achtet, sondern auch deren politische und verfassungsrechtliche Struktur ,einschließlich der regionalen und kommunalen Selbstverwaltung“.

- Das Subsidiaritätsprinzip wird im Sinne der Regionen dahingehend präzisiert, dass für das Tätigwerden der Union nicht nur Maßnahmen auf der Ebene der Mitgliedstaaten

8 Vgl. Erklärung (Nr. 20) zur Erweiterung der Europäischen Union in der Schlussakte des Vertrags von Nizza. Berücksichtigt wurden ausschließlich Staaten, mit denen die Union bereits Beitrittsverhandlungen aufgenommen hatte.

9 Die Methode der offenen Koordinierung wurde mit dem Lissabon-Prozess im Jahr 2000 eingeführt und enthält zwischenstaatliche Vereinbarungen, die aus Sicht einiger Regionen, insbesondere der deutschen Länder, insofern nachteilige Folgen haben, als die nationalen Regierungen Vereinbarungen treffen, für deren Umsetzung und auch Finanzierung in vielen Fällen die Regionen gerade stehen müssen, die an den Beratungen gar nicht beteiligt waren (vgl. Große Hüttmann 2004). 
„nicht ausreichend“ erreicht werden können. Nunmehr wird hier explizit neben der zentralen auch die regionale und lokale Ebene genannt (Art. I-11). Das Prinzip der Verhältnismäßigkeit wurde ebenso präzisiert.

- Das zum Schutz der Subsidiarität eingerichtete Frühwarnsystem ermächtigt beide Kammern der nationalen Parlamente - im Falle Deutschlands also auch den Bundesrat - zur Intervention. Stellungnahmen von Regionen und Kommunen sind vor Erlass von EURegelungen zu berücksichtigen (Protokolle zur Subsidiarität und zu den nationalen Parlamenten).

- Die Zweiten Kammern der nationalen Parlamente erhalten ein Klagerecht (Protokoll zu den nationalen Parlamenten). Ein Klagerecht für einzelne Regionen wurde nicht vorgesehen.

- Dem Wunsch der Regionen nach einer klareren Kompetenzordnung entspricht der Verfassungsvertrag durch die Systematisierung der Zuständigkeiten der Union in den Art.I-11 bis I-17.

- Dem Ausschuss der Regionen wird in Art. III-365 eine Klagerecht vor dem Gerichtshof zur Wahrung seiner Rechte eingeräumt. ${ }^{10}$

Obgleich an mehreren Stellen Forderungen der Regionen in den Verfassungstext aufgenommen worden sind, bleibt die Beurteilung des Erreichten aus der Sicht der Regionen und insbesondere der deutschen Länder, die sehr ambitionierte Ziele gesteckt hatten, ambivalent: Mag man das Frühwarnsystem als Chance zur Verhinderung unliebsamer Entwicklungen aus Sicht der Regionen begreifen, so wird den übrigen Bestimmungen der Charakter von „Verfassungslyrik“ zugeschrieben. Dass nicht mehr an materiellen Kompetenzen und Einspruchsmöglichkeiten erreicht wurde, wird darauf zurückgeführt, dass die europäischen Partner nicht gewillt waren, ihre Regionen substanziell aufzuwerten, sodass die deutschen Länder mit ihren weitgehenden Forderungen isoliert waren (Bauer 2004: 463).

Als Zwischenbilanz ist festzuhalten: Das EU-Primärrecht hat von den Regionen Kenntnis genommen. In der institutionellen Architektur der EU haben sie insbesondere seit dem Vertrag von Maastricht an Bedeutung gewonnen. Das Subsidiaritätsprinzip wurde zum Schutz regionaler Interessen immer mehr verfeinert. In Form eines Ausschusses können die Regionen beratend an der Entscheidungsfindung mitwirken. Mitgliedstaaten können sich durch Vertreter der Regionen im Rat vertreten lassen. Aber die Aufwertung der Regionen blieb eng begrenzt. Von einer Föderalisierung, wie sie den deutschen Ländern vorschwebt, ist die EU noch meilenweit entfernt. Ein wichtiger Grund liegt in der Heterogenität der Regionen selbst. Gebietseinheiten mit Gesetzgebungskompetenzen auf der einen Seite stehen Einheiten mit deutlich weniger Rechten gegenüber. Auch haben die unitarischen und Zentralstaaten wenig Interesse gezeigt, ihre Regionen materiell aufzuwerten.

Trotz der genannten Fortschritte aus Sicht der Regionen bleibt die EU ein Spiegelbild des Staatsaufbaus ihrer Mitgliedstaaten: Da wo die Staaten sich intern einer Entwicklung

10 In der Literatur wird dieses Klagerecht allerdings in seiner Bedeutung relativiert, da die Interessenlage im Ausschuss sehr heterogen ist und eine zielgerichtete und homogene Interessenvertretung erschwert. 
zur Regionalisierung oder gar Föderalisierung entgegenstellen, hat sich auch in der EU der Widerstand gegen eine zu weit reichende Aufwertung der Regionen formiert. Regionen, die wie die deutschen Länder die ,dritte Ebene“ in der EU mit ambitionierten Schritten stärken wollten, blieben zumeist isoliert. Regionen mit Gesetzgebungskompetenzen (Deutschland, Belgien, Österreich) können zwar mit Befriedigung zur Kenntnis nehmen, dass dem Ausschuss der Regionen nach dem Verfassungsvertrag ein Klagerecht eingeräumt werden soll, doch sind diese Regionen mit dem Ausschuss aufgrund seiner Zusammensetzung wenig zufrieden. Eine Stärkung des Ausschusses ist daher für sie durchaus zweischneidig, weil neben Vertretern der Kommunen auch Regionen vertreten sind, die über wenig Eigenständigkeit verfügen und erst recht keinen Staatscharakter aufweisen. Wenn also der Bedeutungszuwachs der Regionen im Vertragswerk der EU nicht überbewertet werden darf, dann ist zu untersuchen, welche Rolle sie im Rahmen der EG-Regionalpolitik spielen.

\section{Die Rolle der Regionen in der EU-Regional- und Strukturpolitik}

Im Rahmen der Regional- und Strukturpolitik steht der EU rund ein Drittel ihrer gesamten Finanzmittel zur Verfügung. Das sind im Jahr 2006 immerhin knapp 38 Mrd. Euro. Um zu klären, ob die Regionalpolitik der EG damit einen Einfluss auf die Stellung der Regionen ausübt, müssen die folgenden Fragen beantwortet werden:

- Welche Rolle spielen Regionen in der übergeordneten Zielsetzung europäischer Regionalpolitik?

- Wie gestaltet sich die Zusammenarbeit zwischen der europäischen, nationalen und regionalen Ebene?

- Wie werden die Gebietseinheiten an der Planung, Erörterung und Umsetzung strukturpolitischer Maßnahmen beteiligt?

- Welchen Einfluss können die Regionen bei der Strukturpolitik im Verlauf des PolitikZyklus vom Agenda-Setting bis zur Implementation ausüben? Inwieweit unterscheiden sich dabei die Verfahren und Einflusschancen, wenn es einerseits um Maßnahmen geht, die im Rahmen von Ziel 1 (vgl. Tabelle 1) vorwiegend aus dem Regionalfonds finanziert werden, und andererseits um Maßnahmen, die aus dem Kohäsionsfonds gefördert werden?

Die europäische Strukturpolitik ist überwiegend regionalpolitisch ausgerichtet: Der Regionalpolitik im engeren Sinne stehen derzeit, d. h. in der Finanzperiode 2000 bis 2006, gut $81 \%$ der gesamten Strukturfonds-Mittel zur Verfügung. Die Strukturpolitik kennt drei Förderziele, wovon Ziel 1 und Ziel 2 regionalpolitisch ausgerichtet sind (vgl. Tabelle 1). 
Tabelle 1: Die Förderziele in der EU-Regionalpolitik ${ }^{11}$

\begin{tabular}{|l|l|}
\hline Ziele & \\
\hline 1 & $\begin{array}{l}\text { Förderzweck: } \\
\text { „Förderung der Entwicklung und der strukturellen Anpassung der Regionen mit Ent- } \\
\text { wicklungsrückstand“ } \\
\text { Förderkriterien: } \\
\text { BIP/Kopf unter 75 \% des EU-Durchschnitts, Gebiete in extremer Randlage, frühere } \\
\text { Ziel 6-Gebiete }\end{array}$ \\
\hline 2 & $\begin{array}{l}\text { Förderzweck: } \\
\text { „Unterstützung der wirtschaftlichen und sozialen Umstellung der Gebiete mit Struk- } \\
\text { turproblemen“ } \\
\text { Förderkriterien: } \\
\text { Arbeitslosenquote, Beschäftigtenquote, Bevölkerungsdichte, hohes Armutsniveau, } \\
\text { schlechte Umweltbedingungen, hohe Kriminalitätsrate, niedriges Bildungsniveau, } \\
\text { Umstrukturierungsprobleme u. ä. }\end{array}$ \\
\hline 3 & $\begin{array}{l}\text { Förderzweck: } \\
\text { „Unterstützung der Anpassung und Modernisierung der Bildungs-, Ausbildungs- und } \\
\text { Beschäftigungspolitiken und -systeme“ } \\
\text { Förderkriterien: } \\
\text { Arbeitslosigkeit, Gender Gap (Benachteiligung von Frauen), Qualifikationen, Armut, } \\
\text { Übereinstimmung mit nationalem Beschäftigungsplan }\end{array}$ \\
\hline
\end{tabular}

Zusätzlich stehen 5,35 \% der Strukturfondsmittel für Gemeinschaftsinitiativen zur Verfügung, von denen INTERREG für die grenzüberschreitende Zusammenarbeit, LEADER für die ländliche Entwicklung und URBAN für Städte ebenfalls regionalpolitisch ausgerichtet sind.

Der Strukturpolitik stehen mehrere Fonds zur Verfügung: Maßnahmen zur Erreichung der Ziele 1 und 2 werden aus dem Regionalfonds, dem Sozialfonds, dem Europäischen Ausgleichs- und Garantiefonds für die Landwirtschaft und dem Finanzinstrument für die Ausrichtung der Fischerei finanziert. Ziel 3 erhält die Gelder aus dem Sozialfonds. Staaten, die ein Bruttosozialprodukt pro Kopf von unter 90 \% des EU-Durchschnitts aufweisen, erhalten eine Förderung aus dem Kohäsionsfonds. Förderfähig sind Maßnahmen in den Bereichen Umwelt und transeuropäische Netze. Im Unterschied zu den zuvor genannten Zielen mitsamt den dazugehörigen Fonds qualifizieren sich beim Kohäsionsfonds ganze Staatsgebiete und werden auch begünstigt. Die Regionen spielen also keine der Ziel 1Förderung vergleichbare Rolle. Bei der EG-Regionalpolitik im engeren Sinne sind zwei Festlegungen von besonderer Bedeutung: erstens die Zweckbestimmung dieser Politik in Artikel 158 des EG-Vertrags und zweitens das im einschlägigen Sekundärrecht (Verordnungen) verankerte Prinzip der ,Partnerschaft“.

11 Vgl. Axt 2000a: $83 \mathrm{ff}$. 
Art. 158 des EG-Vertrags legt als grundlegendes Ziel für den wirtschaftlichen und sozialen Zusammenhalt in der Gemeinschaft fest: „Die Gemeinschaft entwickelt und verfolgt weiterhin ihre Politik zur Stärkung ihres wirtschaftlichen und sozialen Zusammenhalts, um eine harmonische Entwicklung der Gemeinschaft als Ganzes zu fördern. Die Gemeinschaft setzt sich insbesondere zum Ziel, die Unterschiede im Entwicklungsstand der verschiedenen Regionen und den Rückstand der am stärksten benachteiligten Gebiete oder Inseln, einschließlich der ländlichen Gebiete, zu verringern“.

Mit dieser Zielbestimmung wird den Regionen eine zentrale Stellung in der Regionalpolitik zugewiesen. Zum einen sollen die Entwicklungsunterschiede zwischen ihnen abgebaut werden und zum anderen werden sie selbst wichtige Akteure in der Gestaltung und Umsetzung dieser Politik.

Mit dem im Sekundärrecht verankerten Prinzip der Partnerschaft wird diese Intention zusätzlich verstärkt. Die Mitgliedstaaten haben dafür zu sorgen, dass alle kompetenten Partner, darunter gerade auch die Regionen, in die verschiedenen Stadien der Programmplanung einbezogen werden. Regionalpolitik ist nicht nur das Betätigungsfeld der Europäischen Kommission und der nationalen Exekutiven. Auch die regionalen Einheiten sind aktiv in diese Politik einbezogen. In Art. 8 der Rahmenverordnung von 1999 heißt es: Die EU-Regionalpolitik ,kommt zustande durch eine enge Konzertierung, nachstehend Partnerschaft genannt, zwischen der Kommission, dem Mitgliedstaat und den Behörden und Stellen, die der Mitgliedstaat im Rahmen seiner einzelstaatlichen Regelungen und seiner einschlägigen Praxis benennt, insbesondere den regionalen und lokalen Behörden [...]. Bei der Bestimmung der repräsentativsten Partner auf nationaler, regionaler, lokaler oder anderer Ebene sorgt der Mitgliedstaat entsprechend den einzelstaatlichen Regelungen und seiner Praxis für eine weitgehende und effiziente Beteiligung aller relevanten Stellen [...]. Die Partnerschaft erstreckt sich auf die Vorbereitung, Finanzierung, Begleitung und Bewertung der Interventionen“. ${ }^{2}$

\section{Der Einfluss von Regionen auf die Gestaltung der Strukturpolitik}

Dass die regionalen Gebietseinheiten an der Planung und Umsetzung der Strukturpolitik beteiligt sind, sagt noch nichts über den Einfluss aus, den sie in den verschiedenen Phasen ausüben können. Der Frage, inwieweit sie nicht nur als verlängerter Arm der zentralen Administration auf regionaler Ebene fungieren, sondern auch selbst eigene Vorstellungen einbringen und durchsetzen können, soll im Folgenden nachgegangen werden. Dazu wird auf das in der Politikwissenschaft gebräuchliche Konzept des Politikzyklus zurückgegriffen, der in der Art einer Längsschnittanalyse die einzelnen Phasen des politischen Prozesses differenziert darstellt. Die Analyse reicht vom Zeitpunkt, da eine Maßnahme erstmals

12 Verordnung (EG) Nr. 1260/1999 des Rates vom 21. Juni 1999 mit allgemeinen Bestimmungen über die Strukturfonds, in: Amtsblatt der Europäischen Gemeinschaften, L 161/1 vom 26.06.99 (http:// www.europa.eu.int/comm/regional_policy/sources/docoffic/official/regulation/content/de/02_pdf/ 00_1_sf_1_de.pdf; Download vom 10.10.05). 
auf die Tagesordnung von Erörterungen („Agenda-setting“) gesetzt wird, über Beratung und Beschlussfassung bis hin zur Umsetzung (,Implementation“). Dabei wird zunächst auf den Politikzyklus eingegangen, der bei der Gestaltung des Politikfelds Strukturpolitik im übergreifenden Rahmen maßgeblich ist. Erst im zweiten Schritt wird eine einzelne Phase, die Programmplanung, herausgegriffen und wiederum in einzelne Politikphasen unterteilt.

Wenn es um die Strukturpolitik im Ganzen geht, sind vor allem die folgenden Phasen von Belang:

- In der ersten Phase werden Vorschläge zur Gestaltung der Strukturpolitik vorgelegt. Diesen Part übernimmt im Wesentlichen die Europäische Kommission, der das Initiativrecht zum Entwurf von Rechtsakten zusteht. Hier geht es um Verordnungen, die für eine mehrjährige Finanzperiode die Strukturpolitik konzipieren. ${ }^{13}$

- Nachfolgend werden die Finanzmittel festgelegt, die der EU während der Finanzperiode für den Bereich der Strukturpolitik zur Verfügung gestellt werden.

- In der dritten Phase geht es um zweierlei: Einerseits müssen die einschlägigen Verordnungen von den dazu ermächtigten Akteuren beschlossen und andererseits die Förderkriterien im Detail bestimmt werden.

- In Phase 4 werden die verfügbaren Fördermittel auf die Empfänger aufgeteilt, indem die Kommission die jeweilige Fördersumme durch die Variation der Pro-Kopf-Förderung differenziert zuteilt.

- In der fünften Phase erfolgt die Programmplanung, in der die Empfänger zunächst ihre Entwicklungspläne entwerfen, diese mit der Kommission abstimmen und in operationalisierte Programme umformen.

- In der letzten Phase schließlich werden die von der Strukturpolitik begünstigten Maßnahmen umgesetzt.

Es interessieren in diesem Zusammenhang vor allem drei Akteure: die Europäische Kommission, die Mitgliedstaaten und die regionalen Gebietseinheiten. Tabelle 2 ist deren Einfluss in den jeweiligen Politikphasen zu entnehmen. Es fällt zweierlei auf: Erstens gestaltet sich der Einfluss der genannten Akteure sehr unterschiedlich in den einzelnen Politikphasen. Während z. B. die Kommission bei der Vorlage der Verordnungsvorschläge die dominierende Rolle innehat, spielt sie bei der Umsetzung strukturpolitischer Maßnahmen fast gar keine Rolle. Zweitens differiert der Einfluss von Kommission, Mitgliedstaaten und Regionen sehr stark, je nachdem ob es sich um die Ziel 1-Förderung (Regionalpolitik), oder aber die Förderung nach dem Kohäsionsfonds (Strukturpolitik im weiteren Sinne) handelt.

Die Analyse kann sich hier auf die Rolle der Regionen beschränken: Bei der Förderung nach dem Kohäsionsfonds spielen die Regionen in keiner Phase des Politikprozesses eine wichtige Rolle. Diese Form der strukturpolitischen Förderung ist „,staatenzentriert“" ${ }^{14}$

13 Gegenwärtig befinden wir uns in der Finanzperiode 2000 bis 2006. Man unterscheidet Rahmen-, Koordinierungs- und Durchführungsverordnungen (vgl. Axt 2000b: 58 ff.). Die Vorschläge für die neue Finanzperiode 2007 bis 2013 hat die Kommission im Juli 2004 vorgelegt (Kommission der Europäischen Gemeinschaften 2004). 
Im Rahmen der Förderung aus dem Kohäsionsfonds sind deshalb keine relevanten Effekte zu erwarten, die sich auf die Stellung der Regionen auswirken und etwa im Sinne einer Regionalisierung der EU wirksam werden.

Bei der regionalpolitischen Förderung im Rahmen von Ziel 1 spielen die Regionen dagegen in vielen Bereichen eine durchaus einflussreiche Rolle. Die Mitgliedstaaten verlieren im Vergleich zur Kohäsionsfondsförderung an Gewicht. Der Kompetenzzuwachs der Kommission geht zulasten der Mitgliedstaaten. Die Regionen können in der Ziel 1-Förderung bei der Vorlage von Rechtsakten, den Finanzverhandlungen, der Verabschiedung von Verordnungen, den Förderkriterien und der Feinverteilung der Finanzmittel keinen Einfluss geltend machen. Bei der Programmplanung und bei der Umsetzung haben sie dazu jedoch reichlich Gelegenheit. Ihr Kompetenzzuwachs geht hier zulasten der Kommission und teilweise auch der Mitgliedstaaten; Letzteres aber nur, wenn es sich um föderale oder stark regionalisierte Staaten handelt. Im Bereich der Ziel 1-Förderung lohnt es sich damit auch, der Frage nachzugehen, ob die europäische Strukturpolitik positiv auf die Regionalisierung der EU einwirkt.

Tabelle 2: Einfluss der Akteure im Verlauf der Politikphasen bei der Ziel 1-Förderung und beim Kohäsionsfonds ${ }^{15}$

\begin{tabular}{|l|l|l|l|l|l|l|}
\hline Politikphasen & \multicolumn{2}{|l|}{ Kommission } & \multicolumn{2}{l|}{ Mitgliedstaaten } & \multicolumn{2}{l|}{ Regionen } \\
\hline & Ziel 1 & $\begin{array}{l}\text { Kohä- } \\
\text { sions- } \\
\text { fonds }\end{array}$ & Ziel 1 & $\begin{array}{l}\text { Kohä- } \\
\text { sions- } \\
\text { fonds }\end{array}$ & Ziel 1 & $\begin{array}{l}\text { Kohä- } \\
\text { sions- } \\
\text { fonds }\end{array}$ \\
\hline $\begin{array}{l}\text { Phase 1: } \\
\text { Agenda Setting }\end{array}$ & stark & stark & schwach & schwach & $\begin{array}{l}\text { unbe- } \\
\text { deutend }\end{array}$ & $\begin{array}{l}\text { unbe- } \\
\text { deutend }\end{array}$ \\
\hline $\begin{array}{l}\text { Phase 2: } \\
\text { Aushandlung des } \\
\text { Finanzrahmen }\end{array}$ & schwach & schwach & stark & stark & $\begin{array}{l}\text { unbe- } \\
\text { deutend }\end{array}$ & $\begin{array}{l}\text { unbe- } \\
\text { deutend }\end{array}$ \\
\hline $\begin{array}{l}\text { Phase 3: } \\
\text { a) Schaffung des } \\
\text { rechtlich-institutio- } \\
\text { nellen Kontexts }\end{array}$ & stark & stark & stark & stark & $\begin{array}{l}\text { unbe- } \\
\text { deutend }\end{array}$ & $\begin{array}{l}\text { unbe- } \\
\text { deutend }\end{array}$ \\
\hline $\begin{array}{l}\text { Phase 3: } \\
\text { b) Festlegung der } \\
\text { Förderkriterien }\end{array}$ & stark & entfällt & schwach & entfällt & $\begin{array}{l}\text { unbe- } \\
\text { deutend }\end{array}$ & entfällt \\
\hline $\begin{array}{l}\text { Phase 4: } \\
\text { Fein- bzw. indikative } \\
\text { Verteilung der Mittela) }\end{array}$ & stark & stark & $\begin{array}{l}\text { unbe- } \\
\text { deutend }\end{array}$ & stark & $\begin{array}{l}\text { unbe- } \\
\text { deutend }\end{array}$ & entfällt \\
\hline
\end{tabular}

14 Zur Unterscheidung von staaten- und regionenzentrierter Förderung in der Strukturpolitik vgl. ausführlich Axt 2002, 2005.

15 Übernommen aus Axt 2002: 214. 
EU-Regionalpolitik und das „Europa der Regionen“

\begin{tabular}{|l|l|l|l|l|l|l|}
\hline Politikphasen & \multicolumn{3}{|l|}{ Kommission } & Mitgliedstaaten & \multicolumn{2}{l|}{ Regionen } \\
\hline & Ziel 1 & $\begin{array}{l}\text { Kohä- } \\
\text { sions- } \\
\text { fonds }\end{array}$ & Ziel 1 & $\begin{array}{l}\text { Kohä- } \\
\text { sions- } \\
\text { fonds }\end{array}$ & Ziel 1 & $\begin{array}{l}\text { Kohä- } \\
\text { sions- } \\
\text { fonds }\end{array}$ \\
\hline $\begin{array}{l}\text { Phase 5: } \\
\text { Programmplanung }\end{array}$ & $\begin{array}{l}\text { mittel bis } \\
\text { stark je } \\
\text { nach Staat }\end{array}$ & stark & $\begin{array}{l}\text { mittel bis } \\
\text { stark je } \\
\text { nach Staat }\end{array}$ & stark & $\begin{array}{l}\text { mittel bis } \\
\text { stark je } \\
\text { nach Staat }\end{array}$ & $\begin{array}{l}\text { unbe- } \\
\text { deutend }\end{array}$ \\
\hline $\begin{array}{l}\text { Phase 6: } \\
\text { Implementation }\end{array}$ & schwach & schwach & $\begin{array}{l}\text { mittel bis } \\
\text { stark je } \\
\text { nach Staat } \\
\text { und Maß- } \\
\text { nahme }\end{array}$ & stark & $\begin{array}{l}\text { mittel bis } \\
\text { stark je } \\
\text { nach Staat } \\
\text { und Maß- } \\
\text { nahme }\end{array}$ & schwach \\
\hline
\end{tabular}

a) Das geschieht in der Finanzperiode 2000-2006 bei Ziel 1 nach Art. 29 der Rahmenverordnung (Verordnung (EG) Nr. 1260/1999 [Vgl. Fußnote 15] und beim Kohäsionsfonds nach Art. 4 und in Anwendung der dort festgehaltenen Kriterien durch Anhang I zur Kohäsionsfonds-Verordnung (Verordnung (EG) 1264/1999).

\section{Die Beteiligung der Regionen in der Politikphase der Programmplanung}

Welchen Einfluss die Regionen bei der Ziel 1-Förderung in der Programmplanung und der Implementation ausüben können, hängt vom Staatsaufbau der jeweiligen Mitgliedstaaten ab. Regionen in föderal organisierten Staaten können deutlich mehr Einfluss auf die Gestaltung regionalpolitischer Maßnahmen nehmen als die Gebietseinheiten in Zentralstaaten.

Tabelle 3: Index regionaler Autonomie in ausgewählten EU-Staaten

\begin{tabular}{|c|c|c|c|c|c|c|}
\hline & $\begin{array}{l}\text { Födera- } \\
\text { lismus }\end{array}$ & $\begin{array}{l}\text { Besondere } \\
\text { territoria- } \\
\text { le Auto- } \\
\text { nomie }\end{array}$ & $\begin{array}{l}\text { Regionale } \\
\text { Ausgaben }\end{array}$ & $\begin{array}{l}\text { Regio- } \\
\text { naler Steu- } \\
\text { eranteil }\end{array}$ & $\begin{array}{l}\text { Beteili- } \\
\text { gung der } \\
\text { Regionen } \\
\text { an zentral- } \\
\text { staatlicher } \\
\text { Politik }\end{array}$ & Summe \\
\hline & $\begin{array}{l}\text { Werte von } \\
0-4\end{array}$ & $\begin{array}{l}\text { Werte von } \\
0-2\end{array}$ & $\begin{array}{l}\text { Werte von } \\
0-2\end{array}$ & $\begin{array}{l}\text { Werte von } \\
0-2\end{array}$ & $\begin{array}{l}\text { Werte von } \\
0-2\end{array}$ & $\begin{array}{l}\text { Werte von } \\
0-12\end{array}$ \\
\hline Deutschland & 4 & 0 & 2 & 2 & 2 & 10 \\
\hline Belgien & 3 & 1 & 1 & 0 & 2 & 7 \\
\hline \multicolumn{7}{|l|}{ Spanien } \\
\hline - Sonderstatut & 3 & 2 & 1 & 1 & 0 & 7 \\
\hline - Normalstatut & 3 & 1 & 1 & 1 & 0 & 6 \\
\hline
\end{tabular}




\begin{tabular}{|c|c|c|c|c|c|c|}
\hline & $\begin{array}{l}\text { Födera- } \\
\text { lismus }\end{array}$ & $\begin{array}{l}\text { Besondere } \\
\text { territoria- } \\
\text { le Auto- } \\
\text { nomie }\end{array}$ & $\begin{array}{l}\text { Regionale } \\
\text { Ausgaben }\end{array}$ & $\begin{array}{l}\text { Regio- } \\
\text { naler Steu- } \\
\text { eranteil }\end{array}$ & $\begin{array}{l}\text { Beteili- } \\
\text { gung der } \\
\text { Regionen } \\
\text { an zentral- } \\
\text { staatlicher } \\
\text { Politik }\end{array}$ & Summe \\
\hline & $\begin{array}{l}\text { Werte von } \\
0-4\end{array}$ & $\begin{array}{l}\text { Werte von } \\
0-2\end{array}$ & $\begin{array}{l}\text { Werte von } \\
0-2\end{array}$ & $\begin{array}{l}\text { Werte von } \\
0-2\end{array}$ & $\begin{array}{l}\text { Werte von } \\
0-2\end{array}$ & $\begin{array}{l}\text { Werte von } \\
0-12\end{array}$ \\
\hline Frankreich & 1 & 0 & 1 & 1 & 0 & 3 \\
\hline $\begin{array}{l}\text { Überseeische } \\
\text { Gebiete }\end{array}$ & 1 & 1 & 1 & 1 & 0 & 4 \\
\hline $\begin{array}{l}\text { Groß- } \\
\text { britannien }\end{array}$ & 0 & 0 & 1 & 1 & 0 & 2 \\
\hline $\begin{array}{l}\text { Schottland, } \\
\text { Wales, } \\
\text { Nordirland }\end{array}$ & 0 & 1 & 1 & 1 & 0 & 3 \\
\hline Italien & 1 & 0 & 0 & 0 & 0 & 1 \\
\hline Griechenland & 0 & 0 & 0 & 0 & 0 & 0 \\
\hline Irland & 0 & 0 & 0 & 0 & 0 & 0 \\
\hline
\end{tabular}

Quelle: Marks 1996: 331. Die Darstellung bezieht sich auf das Jahr 1989. Aktuellere Befunde für die Mitgliedstaaten der EU-15 finden sich in Abschnitt 7.

Als Ergebnis lässt sich festhalten: In Ländern wie Deutschland, Belgien und Spanien, die als „stark föderal“ bzw. ,föderal“ eingestuft werden, verfügen die Regionen über einen vergleichsweise hohen Grad an regionaler Autonomie. Am anderen Ende der Skala stehen Einheitsstaaten wie Griechenland, Irland und Italien. Andere Einheitsstaaten wie Frankreich und Großbritannien räumen den Regionen begrenzte Mitwirkungsmöglichkeiten ein. Das betrifft insbesondere eine begrenzte Finanzautonomie in Form der Erhebung eigener Einnahmen und deren Verwendung. Dagegen gewähren diese Zentralstaaten ihren Regionen keinen Einfluss auf die Gestaltung der nationalstaatlichen Politik.

Die den Regionen gewährte Autonomie hat Folgen für den Einfluss, den sie in den einzelnen Politikphasen der Programmplanung im Bereich der Ziel 1-Förderung in den ausgewählten Mitgliedstaaten ausüben können. Der Reihenfolge nach ist die Gestaltungskraft der Regionen in folgenden Staaten am stärksten: Deutschland, Belgien, Spanien, Italien, Frankreich, Griechenland, Irland und Großbritannien (Tabelle 4). 
Tabelle 4: Politischer Einfluss von Regionen in der Programmplanung der Ziel 1-Förderung

\begin{tabular}{|l|l|l|l|l|}
\hline & $\begin{array}{l}\text { Entwurf der } \\
\text { Entwicklungs- } \\
\text { pläne }\end{array}$ & $\begin{array}{l}\text { Gemeinschaft- } \\
\text { liche Förder- } \\
\text { konzepte }\end{array}$ & $\begin{array}{l}\text { Operationelle } \\
\text { Programme }\end{array}$ & $\begin{array}{l}\text { Implemen- } \\
\text { tation }\end{array}$ \\
\hline Deutschland & stark & stark $^{\text {a) }}$ & stark & stark \\
\hline Belgien & mittel & stark & stark & mittel-stark \\
\hline Spanien & mittel-stark & schwach & stark & stark \\
\hline Italien & schwach-mittel & schwach & schwach-mittel & schwach-mittel \\
\hline Frankreich & schwach & unbedeutend & schwach & schwach \\
\hline Griechenland & schwach & unbedeutend & schwach & schwach \\
\hline Irland & unbedeutend & unbedeutend & unbedeutend & unbedeutend \\
\hline Großbritannien & unbedeutend & unbedeutend & unbedeutend & unbedeutend \\
\hline
\end{tabular}

a) Die Charakterisierung des Ländereinflusses bei der Abstimmung des Gemeinschaftlichen Förderkonzepts durch Marks als „stark" erscheint problematisch, weil hier lediglich Bund und Kommission agieren, die Länder aber nicht beteiligt sind.

Quelle: Marks 1996: 332. Die Ergebnisse beziehen sich auf die Finanzperiode 1989-1993, aktuellere Entwicklungen werden in Abschnitt 7 berïcksichtigt.

Am Beispiel Deutschlands lässt sich aufzeigen, in welcher Weise die Regionen (Länder) an der Programmplanung im Bereich der Regionalpolitik beteiligt sind. ${ }^{16}$ Der Einfluss der Länder in der Programmplanung muss generell als stark eingeschätzt werden. Bei der Entwicklung der regionalen Entwicklungspläne und der operationellen Programme sowie der Umsetzung haben die Länder eine starke Position inne. Lediglich bei der Abstimmung des gemeinschaftlichen Förderkonzepts können sie nicht mitwirken. Hier agieren Bund und Europäische Kommission. Diese Verhandlungen zwischen Mitgliedstaaten und Kommission finden hinter verschlossenen Türen statt, weshalb auch Marks einräumt, dass hierbei der Einfluss ,,der an den Verhandlungen beteiligten Akteure zu Lasten der Nichtteilnehmer" erhöht wird (1996: 324).

16 Ursprünglich war im Grundgesetz verankert, dass die regionale Wirtschaftsförderung ausschließlich in der Zuständigkeit der Länder liegen sollte. Durch die Etablierung der so genannten „Gemeinschaftsaufgaben“" (Art. 91a GG) wirkt der Bund bei der Verbesserung der regionalen Wirtschaftsstruktur mit. Der Bund konnte die Länder dazu bewegen, auf die ausschließliche Landeszuständigkeit zumindest teilweise zu verzichten, indem vereinbart wurde, dass er die „Hälfte der Ausgaben in jedem Land“" trägt (Art. 91a, 3 GG). Aufgrund der geänderten Bestimmungen im Grundgesetz erging am 6. Oktober 1969 das Gesetz über die „Gemeinschaftsaufgabe Verbesserung der regionalen Wirtschaftsstruktur". 
Heinz-Jürgen Axt

\section{Widersprüchliche Regionalisierungstendenzen in zentralisierten Mitgliedstaaten der} $E U$

Am Beispiel ausgewählter zentralisierter Mitgliedstaaten der EU-15 kann aufgezeigt werden, dass einerseits Regionalisierungsimpulse der EU, insbesondere der Regionalpolitik, Tendenzen zur Dezentralisierung in diesen Staaten begünstigt haben. Andererseits haben es diese Staaten verstanden, diese Entwicklung einzugrenzen und zu verhindern, dass ihre Position geschwächt wurde. In vielen Fällen hat sich der Zentralismus als immer weniger geeignet gezeigt, die Wettbewerbsfähigkeit der Wirtschaft, die Verbesserung der Standortbedingungen, die Optimierung von Verwaltungen, den Abbau sozioökonomischer Disparitäten und den Drang nach Partizipation, also die Mobilisierung des endogenen Potenzials voranzubringen. Dekonzentrations- und Dezentralisierungsentwicklungen waren die Konsequenz. Die staats- und verfassungsrechtliche Aufwertung der Regionen sowie ihre finanzielle Ausstattung blieben jedoch begrenzt.

\section{a) Frankreich}

Frankreich hat 1982 eine Dezentralisierungsreform erlebt, die allerdings keinen Abschied vom Zentralismus implizierte, denn dieser steht noch immer gemäß der vorherrschenden Staatsdoktrin für die „Einheit der Nation“, wobei Letztere nicht den Gegensatz zur Europaorientierung darstellt, sondern im Gegenteil Europa als eine Hülle zur Bewahrung der Nation gesehen wird (Axt 1999). Heute stellt sich Frankreich auf der subnationalen Ebene dreigeteilt dar: 36.763 Kommunen, stehen 100 Departements und 26 Regionen (22 im Mutterland und 4 in Übersee) gegenüber. Diese stehen in keinem hierarchischen Verhältnis zueinander, vielmehr sind sie in Bezug auf die Kompetenzverteilung horizontal angeordnet. Die Region hat demgemäß keine Weisungsbefugnis gegenüber Departements und Kommunen (Zimmermann-Steinhart 2004: 233). Dieser Umstand hat die Konkurrenz der subnationalen Einheiten untereinander gefördert, was zum einen die Regionen geschwächt und zum anderen den Zentralstaat gestärkt hat (Hoffmann-Martinot 1999).

Die Dezentralisierungsreform von 1982 umfasste die folgenden wichtigen Neuerungen: Den Regionen wurde der Status einer Gebietskörperschaft zuerkannt, als Chefs der Regionalverwaltung wurden die Präsidenten der Regionalverwaltungen etabliert, wobei Letztere vom Volk gewählt werden, und schließlich wurden Kompetenzen einschließlich finanzieller Mittel und Personalressourcen delegiert. Seit 1982 sind die Regionen für die regionale Wirtschaftsentwicklung, die Raumplanung und die berufliche Aus- und Weiterbildung zuständig. Insbesondere mit der Zuweisung von Befugnissen im Bereich der Raumplanung und der Regionalentwicklung an die Regionen ist man in Frankreich dem Drängen der EU-Regionalpolitik nach einer Aufwertung der regionalen Akteure entgegengekommen. Die Kommission findet mithin regionale Kooperationspartner, wenn es insbesondere nach dem Partnerschaftsprinzip um die Programmplanung geht. 
Allerdings wurde die Aufwertung der Region sogleich dadurch wieder eingeschränkt, dass den Regionalpräsidenten und ihren Verwaltungen die Regionalpräfekte gegenübergestellt wurden, die als Vertreter des Zentralstaates gemeinsam mit der Region die regionalen Entwicklungspläne und Raumordnungsmaßnahmen beschließen (Hoffmann-Martinot 1999). Mithin ist weiterhin der Zentralstaat ein „dominierender Akteur auf der dezentralen Ebene" (Uterwedde 2000: 165). Wenn es um die gesamtstaatliche Gesetzgebung geht, haben die Regionen keine institutionalisierten Mitwirkungsrechte. ${ }^{17}$ Bei der Formulierung der französischen EU-Politik haben die Regionen keine Mitbestimmungsmöglichkeiten. Das betrifft auch Fragen der Neuordnung der Strukturpolitik, die die Regionen direkt angehen. Der nationale Raumordnungsplan ist der regionalen Politik übergeordnet. Seit Sommer 2002 werden allerdings EU-Subventionen nicht mehr über den Zentralstaat, sondern direkt an die Regionen geleitet. Die finanziellen und personellen Ressourcen der Regionen werden als "sehr bescheiden“ charakterisiert, wenngleich sie gegenüber der Zeit vor 1982 explosionsartig angestiegen sind. ${ }^{18}$ Das kumulierte Ausgabenvolumen aller Gebietskörperschaften betrug 1998 etwa die Hälfte des zentralstaatlichen Haushalts. Die mit der EU-Strukturpolitik verbundene Idee der Partnerschaft hat mithin ,in Frankreich nur wenig Wirkung gezeigt“ (Uterwedde 2000: 173). Verantwortlich dafür ist das Misstrauen der zentralstaatlichen Akteure gegenüber der Herausbildung einer regionalen Gegenmacht. Mit der Dezentralisierungsreform war also weder ein Abschied vom Zentralismus verbunden, noch war man in Frankreich bereit, sich in ein „Brüsseler ,Europa der Regionen'“ einzugliedern (Auphan/Brücher 2005: 7).

\section{b) Vereinigtes Königreich}

Das Vereinigte Königreich - bislang einer der am meisten zentralisierten Staaten - hat seit dem Wahlsieg der Labour Party 1997 einen Dezentralisierungsprozess eingeleitet, der als „devolution“, d. h. als Delegation zentralstaatlicher Macht an regionale Einheiten gekennzeichnet ist. In Schottland, Wales und Nordirland wurden Parlamente und in Groß-London eine Behörde sowie in den anderen acht Regionen Englands regionale Entwicklungsagenturen eingerichtet. ${ }^{19}$ Die Dezentralisierung fand in asymmetrischer Weise statt, indem sie den einzelnen Einheiten unterschiedliche Kompetenzen zukommen ließ. Sie beruhte auf der Erfahrung, dass das überzentralisierte britische System Effizienzmängel aufwies, dass bei der Bewältigung der Regionalentwicklung der Zentralstaat überfordert war, und dass man sich den von der EU ausgehenden Regionalisierungsimpulsen stellen musste (Jeffery/Palmer 2000). Das Verfassungsprinzip der letztendlich bestimmenden Souveräni-

17 Das schließt freilich nicht aus, dass informelle Formen der Kooperation durchaus zum Vorteil der Regionen entstehen und sich ein faktisches Mischsystem der Politikverflechtung herausbildet.

18 Aus diesem Grund wird die Verwaltung der Regionen auch als eher ,projektorientiert“ und "schlank“ gekennzeichnet. Vgl. Uterwedde 2000: 169.

19 Diese Einheiten sind mit den NUTS I-Einheiten identisch. 
tät des Parlaments machte es unmöglich, den Gebietseinheiten dauerhaft Kompetenzen zu übertragen und diese auch verfassungsrechtlich festzuschreiben. Das Parlament könnte also in Zukunft die Übertragung der Kompetenzen wieder rückgängig machen.

Die wirtschaftliche Entwicklung und im Falle Schottlands auch die Raumplanung sind neben anderen Zuständigkeiten auf die Gebietseinheiten in Wales, Schottland, Nordirland und die acht Regionalen Entwicklungsagenturen (Regional Development Agencies, RDA) übertragen worden. Die RDA, die mehrheitlich von Vertretern des regionalen privaten Sektors geleitet werden, unterstehen dem für Umwelt, Verkehr und Regionen zuständigen Minister auf Zentralebene. Die den subnationalen Einheiten zugewiesenen Mittel werden als ,bescheiden“ charakterisiert (Jeffery/Palmer 2000: 334). Wenn es um Fragen der EUPolitik geht, dann können sich die Verantwortlichen auf zentraler und regionaler Ebene in Form von so genannten Konkordaten verständigen. Den regionalen Einheiten ist die Verantwortung für die europäische Strukturpolitik in ihren Territorien und für die Ernennung ihrer Vertreter in den Ausschuss der Regionen zugesprochen worden. In EU-Angelegenheiten informiert die Regierung in London auch die Regionaleinheiten und berücksichtigt deren Stellungnahmen. Auch wenn die Devolution als ein noch nicht abgeschlossener Prozess zu betrachten ist, der Entwicklungspotenzial besitzt, so sollte nicht übersehen werden, dass die formale Stellung der Gebietseinheiten schwach ist, dass die Kompetenzübertragung im Konfliktfall rückgängig gemacht werden kann, und dass bislang eher pragmatische Korrekturen an einem sich als ineffizient erweisenden überzentralisierten System vorgenommen worden sind. Bereits jetzt von einem grundsätzlichen Kurswechsel hin zur Regionalisierung, oder gar Föderalisierung zu sprechen, dürfte verfrüht sein.

\section{c) Griechenland}

Das Staatsgebiet im unitarisch geprägten Griechenland ist heute in 13 Regionen unterteilt, die ihre Entstehung und die ihnen zugeteilten Kompetenzen nicht nur der Einsicht verdanken, dass überzentralisierte Verwaltungssysteme unzureichende Ergebnisse erbringen, sondern dass auch die gerade im Falle Griechenlands üppige Förderung aus den EUStrukturfonds der Stärkung regionaler Verwaltungsstrukturen bedarf. Gleichwohl hat die in diesem Kontext ausgelöste Regionalisierung das ,unitarische Prinzip“ als zentrale Ordnungsfunktion von Staat und Verwaltung nicht gefährdet und ,bis heute jeden Föderalisierungsversuch in diesem Land erfolgreich blockiert" (Wenturis 2000: 190).

Unter der Herrschaft von Friedrich Otto von Bayern wurde 1835 das griechische Territorium in 10 Präfekturen (Nomoi) aufgeteilt, seit der Verfassung von 1975 kommt deren Zahl auf 54. An der Spitze der Nomoi, die keine Rechtspersönlichkeit aufweisen, stehen Nomarchen, die die Zentralregierung auf regionaler Ebene vertreten. Traditionell wurde der Nomarch durch die jeweilige Regierung berufen und entlassen, seit 1994 wird er durch die Wählerschaft in den einzelnen Nomoi gewählt. Die Partizipationsmöglichkeiten der Bürger in der regionalen Selbstverwaltung wurden damit erweitert. Nach der Verwaltungsreform von 1986 gehören zu den Kompetenzen der Nomoi u. a. die demokratische 
Planung, die soziale Fürsorge, das Gesundheitswesen, der Arbeitsmarkt, die Industrie, der Handel und das Erziehungswesen.

Die massive Ausweitung der strukturpolitischen Begünstigung Griechenlands durch die 1985 von der EG beschlossenen „Integrierten Mittelmeerprogramme“ (IMP) veranlasste die griechische Regierung 1986 zu einer Verwaltungsreform, um die Fördermittel effektiver administrieren zu können (Axt 2000a: 61 f.). Das griechische Staatsgebiet wurde nunmehr in 13 Regionen („Periferies“) aufgeteilt. Sie sind die erste Stufe der,,peripheren Selbstverwaltung“, während die Nomoi deren zweite Stufe bilden. Die Regionen stellen in Griechenland die NUTS II-Einheiten dar. Sie besitzen keinen Verfassungsrang, d. h. sie beruhen auf gesetzlicher Grundlage und können durch ein entsprechendes Gesetz auch wieder abgeschafft werden. Ein Organ, das es den Regionen ermöglichen würde, an der Gestaltung der nationalen Politik mitzuwirken, fehlt. Aus diesem Grund wird die Zusammenarbeit der Regionen untereinander erheblich erschwert.

Die Regionen werden von einem Regionalsekretär geleitet, der von der Regierung in Athen ernannt wird, er agiert also als Vertreter der Zentralregierung in der Region. Einem Regionalrat, der sich zusammensetzt aus dem Regionalsekretär, den Nomarchen, den Vorsitzenden der Selbstverwaltung in den Nomoi und Vertretern der Stadt- und Kommunalverbände, obliegen die Aufgaben der Regionalplanung und der Verteilung von Finanzmitteln. Darin sind also die Akteure versammelt, die in der Phase der Programmplanung der EU-Strukturpolitik gemeinsam mit der Zentralregierung die regionalen Entwicklungspläne zu entwerfen und die bewilligten Fördermittel zu verteilen und zu verwalten haben. Für diese Zwecke steht seit 1994 eine „Kasse für Regionale Entwicklung“ zur Verfügung, die von einem Verwaltungsrat geleitet wird, deren Vorsitzender der Regionalsekretär ist. Dies unterstreicht die enge Verklammerung der regionalen Verwaltung mit den Verantwortlichen auf zentralstaatlicher Ebene. Mithin können die Regionen als „Transmissionsriemen“ zur Verwaltung und Verteilung der EU-Strukturfondsmittel verstanden werden (Wenturis 2000: 192; Heinelt 1996).

\section{d) Polen}

In allen Beitrittsländern begann der Prozess der Dezentralisierung - als Gegenbewegung zum Zentralismus des sozialistischen Systems - unmittelbar nach der Wende von 1989/ 90. Oftmals ging es aber mehr um die kommunale als um die regionale Ebene. In einer ersten Euphorie wurden die Kommunen gegenüber den Regionen bevorzugt, zumal Letztere oftmals im Verdacht standen, die Stützen des alten Systems und deren Kader zu sein (Dieringer 2005: 484).

Die NUTS I-III-Einheiten sind mittlerweile in allen neuen Mitgliedstaaten ausgewiesen. Da sich diese aber oftmals als wenig leistungsfähig erwiesen haben, ist die Europäische Kommission dazu übergegangen, die europäischen Strukturfondsmittel den Zentralstaaten zu überantworten (Keating/Hughes 2003). Ágh spricht in diesem Zusammenhang vom Paradox einer ,centralization of decentralization“ (2003: 125). 
Polen kann als Vorreiter der regionalen Neugliederung in Mittel- und Osteuropa gesehen werden (Olewinska 2004). Wie in den anderen Beitrittsländern ist auch in Polen die NUTS I-Ebene mit dem gesamtstaatlichen Territorium identisch. Darunter finden sich auf NUTS II-Ebene 16 Einheiten. Der Abbau des polnischen Zentralismus begann mit dem Gesetz über die kommunale Selbstverwaltung im Jahr 1990 (Gorzelak 1999; Bondyra/Czchor 1999). 1998 wurde das Gesetz über die grundlegende dreistufige Territorialgliederung des Staates beschlossen, das die Rechte und Aufgaben der Regionen (Woiwodschaften) fixierte.

Dass Polen trotz dieser Neuerungen weiterhin als unitarischer Staat zu betrachten ist, lag nicht nur an interessierten Akteuren auf zentraler Ebene, sondern interessanterweise fanden sich auch in der Bevölkerung viele Reformgegner, die als Folge der Regionalisierung die Einheit des Staates gefährdet sahen (Bokajlo 2000: 343). Deshalb mussten bei den Regionalisierungsbestrebungen gravierende Einschränkungen berücksichtigt werden: Die Woiwodschaftsversammlung darf keine eigenständige Außenpolitik betreiben, keine eigene Polizei ins Leben rufen und keine Gesetze oder Steuern beschließen. Als Vertreter der Zentralregierung fungiert der Woiwode, der Beschlüsse der Versammlung bis zur gerichtlichen Überprüfung aussetzen kann. In den Woiwodschaften existiert eine Doppelstruktur von Staats- und Selbstverwaltung.

$\mathrm{Zu}$ den Kompetenzen der Woiwodschaften gehört $\mathrm{u}$. a. die Regionalentwicklung. Dabei müssen die Ziele und Interessen staatlicher Politik berücksichtigt werden, sodass also eine ,enge Kooperation zwischen Zentralstaat und Woiwodschaften über das Regierungszentrum für Strategische Studien“ praktiziert wird (Bokajlo 2000: 348). Die Woiwodschaften besitzen keine Verfassungsautonomie, an der Gestaltung der staatlichen Politik nehmen sie nicht teil. Mit ihren Gestaltungsmöglichkeiten im Bereich der Regionalpolitik können die Woiwodschaften effektiv bei der Planung und Umsetzung der Fördermittel aus den EU-Strukturfonds mitwirken.

\section{e) Ungarn}

Ungarns Dezentralisierung nach dem Zusammenbruch des Sozialismus bestand vorrangig in einer Aufwertung der kommunalen Ebene, der gegenüber der regionalen Ebene deutlich weniger Selbstbestimmungsrechte eingeräumt wurden. Die Kommunen machten sich gegen die Institutionalisierung der Regionen stark (Ágh 2003). Nur die Kommunen, nicht aber die regionalen Einheiten der Komitate erhielten Verfassungsrang. Das führte zu einer inkohärenten Struktur: Die Kommunen waren oftmals überlastet, während die Komitate, die immerhin mit einer vom Volk gewählten legislativen Körperschaft ausgestattet sind, „kaum über nennenswerte Funktionen“ verfügen (Dieringer 2000: 371).

Im Bereich der Regionalentwicklung besitzen die Komitate entscheidende Kompetenzen, die freilich die Vorgaben des nationalen Entwicklungsplanes zu berücksichtigen haben. Andererseits sind die Entwicklungsstrategien der Komitate Grundlage für die nationalen Pläne. 1999 kam es - durchaus in Perspektive des angestrebten Beitritts zur EU - zu einer regionalen Neugliederung: Jeweils drei der insgesamt 18 Komitate wurden zu Regionen zusammengefasst. Die 7 neuen Regionen wurden als NUTS II-Einheiten 
klassifiziert, während die Komitate die NUTS III-Ebene darstellen. Bereits in ihrer Antwort auf das ungarische Beitrittsgesuch konnte die Europäische Kommission 1997 konstatieren, dass die Gesetzgebung des Landes den Vorgaben im Bereich der europäischen Regionalpolitik entspricht (Horváth 1999: 126; Bende-Szabó 1999).

\section{Fazit: Regionalisierung wider Willen?}

In der politikwissenschaftlichen Literatur wird die Strukturpolitik oft als wesentlicher Motor zur Veränderung des Charakters der europäischen Integration identifiziert. Etwas vereinfacht kann man drei unterschiedliche Gruppen ausmachen (Axt 2000a: 163 ff.): Eine erste Gruppe fragt danach, ob Strukturpolitik den allgemeinen Integrationsprozess befördert. Dabei wird u. a. daran erinnert, dass mit strukturpolitischen Begünstigungen oftmals zögerliche Mitgliedstaaten dazu gebracht werden konnten, Integrationsfortschritte zu akzeptieren (,side payments“ u. ä.). ${ }^{20}$ Eine zweite Gruppe konzentriert sich auf die Politikinhalte (,policy“) und auf die Frage, ob diese den sich stellenden Herausforderungen angemessen begegnen. ${ }^{21}$ Eine dritte Gruppierung schließlich ist von besonderem Interesse, weil ihre Vertreter vor allem nachzeichnen wollen, in welcher Weise die europäische Strukturpolitik zur Veränderung von Governance im Rahmen der EU beiträgt. Dabei geht es immer auch um die Frage, welche Rolle der regionalen Ebene beigemessen wird und ob durch Strukturpolitik die Regionen aufgewertet werden. ${ }^{22}$

Die Frage, ob die europäische Strukturpolitik zu einem „Europa der Regionen“ führt, lässt sich sicher nicht abschließend behandeln. Aufgrund der zuvor angestellten Untersuchungen lassen sich jedoch einige Effekte genauer identifizieren und es lässt sich eingrenzen, in welchem Umfang es zu einer Regionalisierung wider Willen kommt. Erfahrungen, die im Rahmen der EU-15 gemacht worden sind, können auf die Beitrittsländer übertragen werden. Die Ergebnisse lassen sich wie folgt zusammenfassen:

Nach verbreiteter Auffassung in Wissenschaft und Politik ist die Mobilisierung und Nutzung des endogenen Potenzials unerlässlich, um sozioökonomische Wachstums- und Modernisierungsprozesse zu beschleunigen. Die Entwicklung der regionalen Gebietseinheiten nützt nicht nur diesen, sondern hat auch positive Effekte für die zentralstaatliche Ebene. Diese Erkenntnisse haben auch zentralistisch organisierte Staaten veranlasst, dem Beispiel der regionalisierten und föderalistischen Staaten zu folgen und die Dezentralisierung ihrer Verwaltungen zu forcieren. Zentralisierte Verwaltungssysteme genügen den Anforderungen der Wirtschaftsförderung, der Raumplanung, des Verkehrs- und Bildungswesens auf regionaler Ebene nicht. Bürokratische Systeme bedürfen einer regionalen Komponente, wenn sie effizient sein wollen.

20 Eine Liste von derartigen ,side payments“, die als politische Kosten von Integration betrachtet werden können, findet sich in: Axt 1997: $923 \mathrm{f}$.

21 Vgl. als Beispiel das Gutachten des Verfassers, das sich mit dem „output“ von Strukturpolitik beschäftigt: Axt 2000b.

22 Exemplarisch für diesen letzten Ansatz vgl. Tömmel 1994, 2002; Marks 1996: 341; Petzold 2004: 452. 
Offensichtlich sind Regionalisierungsprozesse in Ergänzung der beschriebenen Entwicklungen durch die EU-Regionalpolitik begünstigt worden. Im Rahmen und als Effekt der europäischen Regionalpolitik lassen sich regionalpolitische Belange durch die Akteure auf regionaler Ebene besser artikulieren und auch gegen den Widerstand der nationalen Ebene wirksamer durchsetzen. Die Europäische Kommission erweist sich als ein wichtiger Bündnispartner für die Akteure der regionalen Gebietseinheiten, soweit es um regionalpolitische Anliegen geht. Sie nimmt Anregungen auf und unterstützt die Regionen auch gegen die Vertreter der nationalen Ebene. Aber auch die Vertreter der Gebietseinheiten sind wichtige Bündnispartner für die Kommission, wenn es z. B. darum geht, den bisherigen, auf den Regionalausgleich fixierten strukturpolitischen Ansatz weiterzuführen. Wie die Verhandlungen zur Agenda 2000, aber auch die aktuellen Diskussionen zur Agenda 2007 für die auf 25 Mitglieder erweiterte EU zeigen, hat diese Allianz bislang Vorschläge abwehren können, die darauf zielen, in den Empfängerstaaten statt des Regionalausgleichs stärker die Wachstumsförderung in das Zentrum der europäischen Strukturpolitik zu stellen und den Mitgliedstaaten in stärkerem Maße die Eigenverantwortung für die Eliminierung regionaler Disparitäten zuzuweisen. ${ }^{23}$ Das von der Kommission in diesem Zusammenhang bevorzugte Instrument sind die von ihr veranstalteten Kohäsionsforen, bei denen gerade die Vertreter der Regionen Gelegenheit erhalten, sich für den Erhalt von strukturpolitischen Transfers zu artikulieren. ${ }^{24}$ Mittlerweile haben sich staatenübergreifende Netzwerke solcher Gebietseinheiten herausgebildet.

Als Folge der Regionalisierung modifizieren sich zwar die Formen der Governance. Eine staats- und verfassungsrechtliche Aufwertung der Regionen kommt allerdings gegen den Willen der Zentralstaaten nicht zustande. Die von der Regionalpolitik ausgehenden Impulse in Richtung einer Regionalisierung sollten nicht überschätzt werden. ${ }^{25}$ Hrbek und Weyand konstatieren: ,In allen Mitgliedstaaten, in denen förmliche oder informelle regionale Beteiligungsverfahren an der Formulierung nationaler EG-Politik existieren, hängen die Einflussmöglichkeiten der Regionen sehr stark von der Kooperationsbereitschaft der

23 Zur Kontrastierung von staaten- und regionenzentrierter Förderung in der Strukturpolitik vgl. ausführlich Axt 2002 und 2005.

24 Für die Agenda 2007 hat die Kommission vorgeschlagen, die Strukturpolitik stärker mit der LissabonStrategie zur Steigerung der Wettbewerbsfähigkeit der EU zu verbinden. Auch dazu hat die Kommission die Regionen um einen aktiven Beitrag gebeten. Am 3. März 2005 führte sie zu diesem Zweck in Brüssel eine Veranstaltung zu dem Thema „, Kohäsion und die Agenda von Lissabon: die Rolle der Regionen“ durch. Die Regionen, so hieß es, hätten viel Erfahrung mit der Förderung einer wettbewerbsfähigeren Wirtschaft. Mit der Unterstützung der Strukturfonds und des Kohäsionsfonds der Europäischen Union arbeiteten sie bereits auf eine Verwirklichung der wesentlichen Aspekte der Lissabon-Agenda hin. Diese Bemühungen müssten fortgesetzt werden (http://europa.eu.int/comm/ regional_policy/sources/docconf/lisbon/index_de.htm, Download von 04.10.05). Vom 10. bis 13. Oktober 2005 veranstaltete die Kommission eine Europäische Woche. Diese „European Week of Regions and Cities" hatte ebenfalls die Aufgabe, die Zukunft der Regionalpolitik zu erörtern.

25 Tömmel konstatiert, dass die „Regionen der EU weit davon entfernt [sind], eine echte ,dritte Ebene' (im Mehrebenensystem) zu konstituieren“, gibt aber zu bedenken, dass die EU ein System ,in statu nascendi“" sei, weshalb sie eine Verstärkung der Rolle der Regionen offensichtlich in Zukunft nicht ausschließen möchte (2002: $56 \mathrm{f}$.). 
jeweiligen Zentralregierung ab. Dies gilt auch dort, wo die Regionen einen Rechtsanspruch auf Information, Konsultation oder gar Mitsprache haben. Denn die Durchsetzbarkeit regionaler Interessen wird wesentlich davon bestimmt, wie stark sich die nationale Regierung in Brüssel für diese einsetzt“ (1994: 95).

Als „Herren der Verträge“ haben die Regierungen der Mitgliedstaaten in den EU-Vertragswerken nur eine begrenzte Aufwertung der Regionen zugelassen. Das ist das Spiegelbild für das, was die Staaten in ihrem inneren Staatsgefüge an Regionalisierung akzeptieren. Auf Drängen der Regionen ist das Subsidiaritätsprinzip immer weiter verfeinert worden. Man hat zugelassen, dass auch Repräsentanten regionaler Exekutiven nationale Minister im Rat vertreten können, und es ist ein Ausschuss der Regionen eingerichtet worden, dem nach dem Verfassungsvertrag auch ein Klagerecht zugestanden werden soll. Doch dürfte eine EU-induzierte Regionalisierung nur dann Chancen haben, wenn die Akteure auf nationaler Ebene zustimmen. Bisher war der Wille dazu offensichtlich nicht vorhanden. Trotz interregionaler Kooperation steckt das „Europa der Regionen“ bis heute noch in seinen Kinderschuhen. Viele haben ihre Ansprüche inzwischen heruntergeschraubt; man spricht lieber vom „Europa mit den Regionen“ (Buchheim 2002: 54). Und mit Bezug auf die deutschen Länder als besonders energische Vertreter regionaler Interessen auf EU-Ebene ist mittlerweile sogar konstatiert worden, dass sich die Strategie vom „Lasst uns rein“ (in das EU-Entscheidungssystem) gewandelt habe zum „Lasst uns allein“, damit innerstaatlich errungene Kompetenzen nicht gefährdet werden (Große Hüttmann 2005: 32). Schließlich ist anzumerken, dass die Kooperation unter den Regionen durch den sehr unterschiedlichen Status erschwert wird, der ihnen im Aufbau der jeweiligen Mitgliedstaaten gewährt wird.

Die Erfahrungen mit den alten Mitgliedstaaten können auf die neuen EU-Staaten übertragen werden. Die im Rahmen der europäischen Regionalpolitik erforderlich gewordene Schaffung regionaler Gebietseinheiten (NUTS) vermehrt die Chancen, dass regionale Entwicklungsbelange stärker - auch gegen die Dominanz der Zentralen - artikuliert werden können. In der Kommission finden die Gebietseinheiten stets einen Allianzpartner. Um in den Genuss europäischer Fördermittel zu gelangen, widersetzen sich die national verantwortlichen Akteure auch nicht der stark auf Regionalausgleich setzenden EUPolitik. Auch die neuen Mitgliedstaaten lassen sich am „goldenen Zügel“ der EU führen. Wenn es allerdings darum geht, den Staatsaufbau zu dezentralisieren und den Regionen größere, auch verfassungsrechtlich abgesicherte Kompetenzen zu übertragen, ist der Einfluss der europäischen Regionalpolitik nicht stark genug, um Zentralstaaten gegen ihren Willen zu einem Kurswechsel zu veranlassen.

Sollten sich die Vorschläge der Kommission zur Gestaltung der europäischen Strukturpolitik für die Jahr von 2007 bis 2013 durchsetzen, dann werden die neuen Mitgliedstaaten immerhin ein Drittel ihrer Förderung aus dem Kohäsionsfonds erhalten (Europäische Kommission 2004; Kommission der Europäischen Gemeinschaften 2004). Das schränkt die Möglichkeiten der regionalen Gebietseinheiten ein, ihre Anliegen eigenständig zu artikulieren und im Politikprozess umzusetzen. Die Regionen spielen bei der Förderung aus dem Kohäsionsfonds keine bedeutende Rolle. 
Schließlich ist daran zu erinnern, dass die eigentliche Intention europäischer Regional- und Strukturpolitik nicht darin besteht, die EU in Richtung eines „Europas der Regionen“ zu entwickeln. Soweit Effekte zur Aufwertung der Regionen zu registrieren sind, handelt es sich eher um Nebeneffekte einer Politik, die in ihrem eigentlichen Kern als Umverteilungspolitik zu identifizieren ist. Was z. B. in Deutschland als Finanzausgleich zwischen den Ländern stattfindet, hat in der EU vornehmlich die Strukturpolitik übernommen: Wohlhabendere Staaten unterstützen die weniger entwickelten Mitglieder, indem sie ihnen Transfers aus den Strukturfonds zukommen lassen. Damit wird der Binnenmarkt mit seinen Charakteristika des Wettbewerbs und der Liberalisierung um das für politisch erforderlich gehaltene Element der ,Solidarität“ ergänzt. Dass man dabei nicht einem einfachen Finanzausgleich zugunsten der wirtschaftsschwächeren Staaten den Vorzug gegeben, sondern ein administrativ aufwändiges System bevorzugt hat, bei dem Mittel an bestimmte Zwecke gebunden, die Regionen einbezogen und der Kommission Kontrollrechte übertragen werden, hat mit dem Wunsch insbesondere der Nettozahler zu tun, eine zweckfremde Verwendung der europäischen Strukturfondsmittel zu unterbinden. ${ }^{26}$

\section{Literatur}

Ágh, Attila, 2003: Anticipatory and Adaptive Europeanization in Hungary, Budapest. Auphan, Etienne/Brücher, Wolfgang, 2005: Frankreichs (De-)Zentralisierung im Kontext der europäischen Integration, in: Geographische Rundschau 9, 4-10.

Axt, Heinz-Jürgen, 1997: Strukturpolitik und Kohäsion in der Europäischen Union: Reform in der Perspektive der Osterweiterung, in: ZPol 3, 885-927.

Axt, Heinz-Jürgen, 1999: Frankreich in der Europäischen Union, in: Marieluise Christadler/Henrik Uterwedde (Hrsg.), Länderbericht Frankreich: Geschichte, Politik, Wirtschaft, Gesellschaft, Opladen, 465-483.

Axt, Heinz-Jürgen, 2000a: EU-Strukturpolitik. Einführung in die Politik des wirtschaftlichen und sozialen Zusammenhalts, Opladen.

Axt, Heinz-Jürgen, 2000b: Solidarität und Wettbewerb - die Reform der EU-Strukturpolitik, Gütersloh.

Axt, Heinz-Jürgen, 2002: Strukturpolitik in der erweiterten EU: statt Regionalausgleich. Wachstum fördern?, in: Beihefte der Konjunkturpolitik. Zeitschrift für angewandte Wirtschaftsforschung. Osterweiterung der EU. 65. Wissenschaftliche Tagung der Arbeitsgemeinschaft deutscher wirtschaftswissenschaftlicher Forschungsinstitute am 25. und 26. April 2002, Berlin, 181-215.

26 Die Kommission wurde - beginnend mit den Integrierten Mittelmeerprogrammen von 1985 und fortgeführt mit der Reform der Strukturpolitik von 1988 - mit der Prüfung der Verwendung der zugeteilten Mittel beauftragt, weil die Nettozahler diese Kontrolle nicht selbst vornehmen konnten (Axt 2000a: $61 \mathrm{ff}$.$) .$ 
Axt, Heinz-Jürgen, 2005: Alter Wein in neuen Schläuchen. Warum die Kommission nicht vom Ziel des Regionalausgleichs in der Strukturpolitik abgeht, in: Ines Hartwig/ Wolfgang Petzold (Hrsg.), Solidarität und Beitragsgerechtigkeit. Die Reform der EUStrukturfonds und die Finanzielle Vorausschau, Baden-Baden (Schriftenreihe des Arbeitskreises Europäische Integration, Bd. 53), 11-37.

Bauer, Michael W., 2004: Der europäische Verfassungsprozess und der Konventsentwurf aus Sicht der deutschen Länder, in: Europäisches Zentrum für Föderalismus-Forschung Tübingen 2004, 453-475.

Bende-Szabó, Gábor, 1999: The intermediate administrative level in Hungary, in: Eric von Breska/Martin Brusis (Hrsg.), Central and Eastern Europe on the Way into the European Union: Reforms of Regional Administration in Bulgaria, the Czech Republic, Estonia, Hungary, Poland and Slovakia, München, 23-41.

Bokajlo, Wieslaw, 2000: Polen - die neuen Woiwodschaften im Europa der Regionen, in: Europäisches Zentrum für Föderalismus-Forschung Tübingen 2000, 340-357.

Bondyra, Krysztof/Czchor, Zbigniew, 1999: Dezentralisierung und Regionalverwaltung in Polen, in: Eric von Breska/Martin Brusis (Hrsg.), Central and Eastern Europe on the Way into the European Union: Reforms of Regional Administration in Bulgaria, the Czech Republic, Estonia, Hungary, Poland and Slovakia, München, 82-96.

Buchheim, Ute, 2002: Regionale Interessenvertretung in Europa. Nordrhein-Westfalen und Thüringen im Strukturvergleich, Opladen.

Dieringer, Jürgen, 2000: Ungarn - Vom „,demokratischen Zentralismus“ zur dezentralisierten Demokratie?, in: Europäisches Zentrum für Föderalismus-Forschung Tübingen 2000, 370-383.

Dieringer, Jürgen, 2005: Demokratisierung, Ökonomisierung und Europäisierung: Dezentralisierungsprozesse in Ostmitteleuropa, in: Südosteuropa 4, 483-499.

Engel, Christian, 1993: Regionen in der EG. Rechtliche Vielfalt und integrationspolitische Rollensuche. Gutachten im Auftrag der Staats- und Senatskanzleien der Länder, Bonn.

Engel, Christian, 1994: Regionen im Netzwerk europäischer Politik, in: Udo Bullmann (Hrsg.), Die Politik der dritten Ebene. Regionen im Europa der Union, Baden-Baden.

Europäische Kommission, 2004: Eine neue Partnerschaft für die Kohäsion. Konvergenz, Wettbewerbsfähigkeit, Kooperation. Dritter Bericht über den wirtschaftlichen und sozialen Zusammenhalt, Luxemburg.

Europäisches Zentrum für Föderalismus-Forschung Tübingen (Hrsg.), 2000: Jahrbuch des Föderalismus 2000. Föderalismus, Subsidiarität und Regionen in Europa, BadenBaden.

Europäisches Zentrum für Föderalismus-Forschung Tübingen (Hrsg.), 2004: Jahrbuch des Föderalismus 2004. Föderalismus, Subsidiarität und Regionen in Europa, BadenBaden

Eurostat Regionen, 1995: Nomenklatur der statistischen Gebietseinheiten, NUTS, März, Luxemburg. 
Föhn, Cornelia, 2003: Der Ausschuss der Regionen - Interessenvertretung der Regionen Europas. Eine Darstellung unter besonderer Berücksichtigung der deutschen Bundesländer, München.

Gorzelak, Grzegorz, 1999: Regional policies and regional capacity-building in Poland, in: Martin Brusis (Hrsg.), Central and Eastern Europe on the Way to the European Union: Regional Policy Making in Bulgaria, the Czech Republic, Estonia, Hungary, Poland and Slovakia, München, 131-159.

Große Hüttmann, Martin, 2004: Die Offene Methode der Koordinierung in der Europäischen Union: Chancen und Risiken eines neuen Steuerungsinstruments aus Sicht der deutschen Länder, in: Europäisches Zentrum für Föderalismus-Forschung Tübingen 2004, 476-488.

Große Hüttmann, Martin, 2005: Wie europafähig ist der deutsche Föderalismus?, in: APuZ, B 13/14, 27-32.

Heinelt, Hubert (Hrsg.), 1996: Politiknetzwerke und europäische Strukturfondsförderung. Ein Vergleich zwischen EU-Mitgliedstaaten, Opladen.

Heller, Wilfried/Aschauer, Wolfgang, 2004: Regionen als Instrumente der Kooperation und Integration, in: Revue des études sud-est européennes, hrsg. v. Académie Roumaine 1 (4), 271-292.

Hoffmann-Martinot, Vincent, 1999: Zentralisierung und Dezentralisierung in Frankreich, in: Marieluise Christadler/Henrik Uterwedde (Hrsg.), Länderbericht Frankreich: Geschichte, Politik, Wirtschaft, Gesellschaft, Opladen, 363-382.

Horváth, Gyula, 1999: Regional and cohesion policy in Hungary, in: Martin Brusis (Hrsg.), Central and Eastern Europe on the Way to the European Union: Regional Policy Making in Bulgaria, the Czech Republic, Estonia, Hungary, Poland and Slovakia, München, 90-130.

Hrbek, Rudolf/Weyand, Sabine, 1994: Betrifft: Das Europa der Regionen. Fakten, Probleme, Perspektiven, München.

Jeffery, Charlie/Palmer, Rosanne, 2000: Das Vereinigte Königreich - Devolution und Verfassungsreform, in: Europäisches Zentrum für Föderalismus-Forschung Tübingen 2000, 321-339.

Jeffery, Charlie/Palmer, Rosanne, 2004: Die britische Devolution und die Wählerschaft: die Wahlen 2003 in Schottland, Wales und Nordirland, in: Europäisches Zentrum für Föderalismus-Forschung Tübingen 2004, 276-289.

Keating, Michael, 1998: Is there a regional level of government in Europe?, in: Patrick Le Galés/Christian Lequesne (Hrsg.), Regions in Europe, London, 11-29.

Keating, Michael/Hughes, James, 2003: The Regional Challenge in Central and Eastern Europe. Territorial Restructuring and European Integration, Bruxelles u. a.

Kommission der Europäischen Gemeinschaften, 2004: Vorschlag für eine Verordnung des Rates mit allgemeinen Bestimmungen über den Europäischen Fonds für regionale Entwicklung, den Europäischen Sozialfonds und den Kohäsionsfonds, SEC (2004) 924, Brüssel, 14.07.04. 
Marks, Gary, 1996: Politikmuster und Einflusslogik in der Strukturpolitik, in: Markus Jachtenfuchs/Beate Kohler-Koch (Hrsg.), Europäische Integration, Opladen, 313343.

Olewinska, Monika, 2004: Dezentralisierungsprozesse in Polen. Von der Grundkonzeption bis zur Realisierung, in: Udo Margedant (Hrsg.), Föderalismus in Europa III, Sankt Augustin (Zukunftsforum Politik, Nr. 64, Konrad Adenauer-Stiftung, http:// www.kas.de/publikationen/2004/5823_dokument.html), 73-97.

Petzold, Wolfgang, 2004: Zur Debatte und den Perspektiven der EU-Strukturpolitik nach 2006, in: Europäisches Zentrum für Föderalismus-Forschung Tübingen 2004, 437-452.

Sturm, Roland/Dieringer, Jürgen, 2004: Theoretische Perspektiven der Europäisierung im Ost-West-Vergleich, in: Europäisches Zentrum für Föderalismus-Forschung Tübingen 2004, 21-35.

Tömmel, Ingeborg, 1994: Staatliche Regulierung und europäische Integration. Die Regionalpolitik der EG und ihre Implementation in Italien, Baden-Baden.

Tömmel, Ingeborg, 2002: Die Regionalpolitik der EU, Systementwicklung durch Politikgestaltung, in: Thomas Conzelmann/Michéle Knodt (Hrsg.), Regionales Europa Europäisierte Regionen, Frankfurt a. M., 39-67.

Uterwedde, Henrik, 2000: Frankreichs gezähmter Jakobinismus: Regionen im zentralisierten Staat, in: Europäisches Zentrum für Föderalismus-Forschung Tübingen 2000, 158-175.

Versammlung der Regionen Europas, 2002: Satzung der Versammlung der Regionen Europas (VRE) (http://www.are-regions-europe.org/PDF/Statutes/D-Statuts-Naples.pdf, Download vom 14.03.05).

Wenturis, Nikolaus, 2000: Griechenland - Historische Hintergründe und aktueller Stand des Dezentralisierungsprozesses, in: Europäisches Zentrum für Föderalismus-Forschung Tübingen 2000, 176-192.

Zimmermann-Steinhart, Petra, 2004: Akt II der französischen Dezentralisierung: Konsequenzen für das politische System und die Gesellschaft, in: Europäisches Zentrum für Föderalismus-Forschung Tübingen 2004, 219-236.

Korrespondenzanschrift:

Prof. Dr. Heinz-Jürgen Axt

Universität Duisburg-Essen

Fachbereich Gesellschaftswissenschaften

Institut für Politikwissenschaft

Jean Monnet-Lehrstuhl

Lotharstr. 63, LF 328

47057 Duisburg

E-Mail: heinz-juergen.axt@uni-due.de 